\title{
Prospects for live attenuated HIV
}

Recent discussion of using live attenuated HIV-1 strains as a vaccine approach for AIDS seems to be raising blood pressures to unusually high levels. The concept of a live attenuated HIV-1 vaccine is understandably difficult to accept. Who would willingly inoculate live HIV, however attenuated, into healthy, uninfected individuals? Who would volunteer? How could you ensure its safety?

Absent from much of the recent discussion and rhetoric is the extent to which many of the more difficult issues can be addressed by traditional scientific method. Specific questions can be asked, experiments performed and answers obtained without exposing humans to potentially risky live vaccine trials. Valuable information can be, and indeed already has been, obtained on whether vaccine protection can be achieved, on the nature of protective immunity, on which live vaccine strains may be more risky and on factors which influence whether vaccine protection is achieved. Progress in these areas is being derived from studies in monkey models, of longterm nonprogressing survivors of HIV-1 infection, and of highly exposed, uninfected humans who may have protective cellular immune responses. Even if the live attenuated vaccine approach is never deemed worthy of human trials, we will learn important lessons for prevention or treatment by other methods.

Considerable attention has been given to misleading or illogical arguments against attempts to develop live atten uated HIV-1 strains for use in trials in human volunteers. These include the often-repeated statements "I never met a retrovirus that didn't cause disease" and "All retroviruses will cause disease eventually." These statements are at best misleading, but closer to simply wrong; there are many examples to the contrary. African green monkeys and sooty mangabey monkeys naturally infected with their own simian immunodeficiency viruses (SIVs) do not develop any SIV-related disease during their natural lifespan. Most rhesus monkeys infected with certain attenuated strains of SIV, such as those missing the nef gene or nef plus vpr genes, maintain undetectable viral burdens without evidence of disease progression for as long as they have been followed.
The oldest nonprogressing survivors have now lived nine years, about $50 \%$ of the lifespan of an aged rhesus monkey.

Considerable attention has also been given to the finding of progression to AIDS in some of the monkeys infected with nef- or nef- plus vpr viruses. For those in the field, these findings have been known for some time. Some of the work has been published ${ }^{1-3}$, some presented and discussed extensively at meetings and workshops, and other results have been discussed informally by those working in the field. The HIV-1 strains that have been suggested for consideration for possible human use, at least by myself and my colleagues, have considerably more genetic information removed from the virus and are based on SIV strains that are much more attenuated than the nef- or nef- plus vpr ${ }^{-}$viruses discussed above. We should not be trapped in a vicious chain of illogic:

attenuated virus $\equiv$ delta nef

delta nef $\equiv$ dangerous

attenuated virus $\equiv$ dangerous

There is a big difference between the attenuated SIV strains that have induced disease in a small percentage of monkey recipients and the much more highly attenuated strains that have been the focus of current research ${ }^{4}$.

W ork so far has shown that a very wide range of attenuation levels can be achieved in SIV simply by varying the number and location of deletion mutations $^{4}$. Not surprisingly, the ability to serve effectively as a vaccine varies inversely with the degree of attenuation. It is easier to protect against vaginal mucosal exposure than against intravenous challenge. We are encouraged that reasonable protection can be achieved against vaginal mucosal exposure even when a highly attenuated vaccine strain is administered systemically more than

\section{Homeless p53 homologues}

The July 1998 issue included two papers presenting new p53 homologues ${ }^{1,2}$. The sequence reference details for the Osada et al. manuscript were inadvertently omitted. The DDBJ and GenBank accession numbers are as follows: p51A, AB016072; and p51B, AB016073.

1. Osada, M. et al. Cloning and functional analysis of human p51, which structurally and functionally resembles p53. Nature Med. 4, 839-843 (1998)

2. Trink, B. et al. A new human p53 homologue. Nature Med. 4, 747-748 (1998) 